\title{
Tensor Product Model-Based Gain Scheduling of a Missile Autopilot*
}

\author{
Tao JIANG ${ }^{\dagger}$ and Defu LIN \\ School of Aerospace Engineering, Beijing Institute of Technology, Beijing 3210, China
}

\begin{abstract}
In this paper, a tensor product model-based gain scheduling technique is utilized to design a pitch-axis autopilot for an air-to-air missile. Firstly, a recent technique for tensor product (TP) model transformation provides a convenient way to transform missile pitch-axis linear-parameter-varying (LPV) model into a convex parameter-varying weighted combination of linear-time-invariant (LTI) systems. This polytopic model is beneficial for convex hull manipulation, so that a large number of linear matrix inequality (LMI) optimization techniques can be applied controller development. This paper presents an alternative LMI technique based on a TP polytopic model, which can optimize the $H_{\infty}$ performance of a closed-loop system with LMI pole constraints. Then, the proposed approach is applied in the design of a simple four-loop gain-scheduled autopilot. Final simulation results indicate the missile autopilot presented has good overall performance and strong robustness, which validates effectiveness of the proposed TP model-based method.
\end{abstract}

Key Words: Missile Autopilot, Tensor Product Model Transmission, Gain-Scheduling, $H_{\infty}$ Optimization, LMI Pole Placement

\section{Introduction}

Missile control problems are a challenging task due to fast and wide parameter variations during operation. As a good method to deal with nonlinear time-varying systems, the advanced LPV technique ${ }^{1-4)}$ has been widely used in missile autopilot design. Compared to conventional gain-scheduling techniques, ${ }^{5-7)}$ LPV techniques can provide the guarantee of stability and performance of a closed-loop system over the whole parameter variation space. Dynamic characteristics of LPV models vary following some time-varying parameters whose values are unknown a priori but can be measured in real-time. In Shamma and Cloutier, ${ }^{2)}$ the concept of a "quasi-LPV" was first introduced, and a state transformation method was proposed to convert nonlinear missile pitch-axis dynamics into a LPV form.

To synthesize such an LPV plant, a discretization scheme ${ }^{8)}$ was proposed to design a missile autopilot, and the controller gains need be updated online to conduct real-time actuation; however, this process imposes significant demand on CPU power. Following $\mathrm{Wu}$, a LPV grid technique ${ }^{4)}$ was introduced, which can relieve the requirement on computer hardware since the main computation is done offline. But it is a repeated process with the set of a denser grid. Other recent LPV techniques, such as the multi-channel linear fractional transformation (LFT)/LPV control method ${ }^{3)}$ and quasilinear parameter-varying polynomial eigenstructure assignment, ${ }^{9)}$ can also provide effective methods to develop a LPV missile autopilot.

In Apkarian and Adams, ${ }^{10)}$ an alternative advanced gainscheduling technique was proposed to obtain a LPV controller via solving a finite number of LMI constraints only at all

(C) 2016 The Japan Society for Aeronautical and Space Sciences

*Received 19 March 2015; final revision received 31 December 2015; accepted for publication 18 January 2016.

†Corresponding author, panpanyunshi@163.com vertices points of parameter variation space. However, this requires models be affinely dependent on the scheduled parameters. Actually, most plants including the missile LPV model do not satisfy this requirement. A novel approach, proposed by Baranyi, ${ }^{11-14)}$ can transform such a LPV model into a convex parameter-varying weighted combination of LTI systems that are capable of finding the TP polytopic approximants of arbitrary accuracy. Due to the advantages of the TP polytopic model for convex hull manipulation, many linear matrix inequality (LMI) design methods ${ }^{15)}$ can be applied in LPV controller development. In spite of a recently proposed technique, much literature has emerged regarding TP model based design, including a 2-DOF aeroelastic system, ${ }^{12,13)} 3$-DOF aeroelastic system, ${ }^{16,17)}$ a TORA system, ${ }^{18)}$ a 3-DOF RC helicopter, ${ }^{19)}$ the vehicle automatic transmission system ${ }^{20)}$ and so on.

In this paper, an alternative TP model based-design approach is utilized to design a LPV state feedback missile autopilot, which optimizes the closed-loop system $H_{\infty}$ performance with pole constraints. This method can guarantee better control of the closed-loop dynamics and avoid the inherent inconveniences of standard $H_{\infty}$ control. $^{21)}$ In the design process, there is no need to select the discrete set of interpolation points in the operating range or complete controller synthesis at each point. The gain-scheduled controller can be obtain in just one computation. Moreover, this proposed technique is more efficient than that of Chumalee and Whidborne ${ }^{22)}$ because more flexible performance specifications can be realized by choosing the proper LMI regions to bound the poles.

The organization of this paper is as follows. A missile LPV model is introduced and transformed into a TP polytopic model in Section 2. In Section 3, our design method based on a TP type plant is proposed to minimize the closed-loop system $H_{\infty}$ norm that enforces pole constraints. Aiming at a specified four-loop missile autopilot structure, 
this technique is applied to complete the controller design. The simulation results are shown and explained in Section 4. Conclusions are stated at the end of this paper.

\section{The Missile Pitch-axis TP Model}

\subsection{The missile pitch-axis quasi-LPV model and per- formance objectives}

The missile pitch-axis quasi-LPV model taken from $\mathrm{Wu}$ et al. ${ }^{4)}$ describes the dynamics of a highly maneuverable air-to-air missile around its pitch axis, which is given by

$$
\begin{aligned}
& {\left[\begin{array}{l}
\dot{\alpha} \\
\dot{q}
\end{array}\right]=\underbrace{\left[\begin{array}{ll}
a_{11} & 1 \\
a_{21} & 0
\end{array}\right]}_{A(\theta)}\left[\begin{array}{l}
\alpha \\
q
\end{array}\right]+\underbrace{\left[\begin{array}{c}
K_{\alpha} M d_{n} \cos (\alpha) \\
K_{q} M^{2} d_{m}
\end{array}\right]}_{B(\theta)} \delta} \\
& {\left[\begin{array}{l}
\eta \\
q
\end{array}\right]=\underbrace{\left[\begin{array}{cc}
c_{11} & 0 \\
0 & 1
\end{array}\right]}_{C(\theta)}\left[\begin{array}{c}
\alpha \\
q
\end{array}\right]+\underbrace{\left[\begin{array}{c}
K_{z} M^{2} d_{n} \\
0
\end{array}\right]}_{D(\theta)} \delta}
\end{aligned}
$$

where

$$
\begin{aligned}
& a_{11}=K_{\alpha} M\left(a_{n} \alpha^{2}+b_{n}|\alpha|+c_{n}\left(-\frac{M}{3}+2\right)\right) \cos (\alpha) \\
& a_{21}=K_{q} M^{2}\left(a_{m} \alpha^{2}+b_{m}|\alpha|+c_{m}\left(-\frac{8 M}{3}-7\right)\right) \\
& c_{11}=K_{z} M^{2}\left(a_{n} \alpha^{2}+b_{n}|\alpha|+c_{n}\left(-\frac{M}{3}+2\right)\right)
\end{aligned}
$$

State vector is angle of attack $\alpha$ and pitch rate $q$. The task for the autopilot is to make sure the missile's normal acceleration $\eta$ tracks varying amplitude reference signal $\eta_{c}$ using the missile's fin deflection angle $\delta$. The numerical constants appearing in Eq. (1) are shown in Table 1.

These coefficients are valid for a missile traveling between Mach numbers 2 and 4 at an altitude of 20,000 ft. Finally, the Mach number dynamics ${ }^{4)}$ are given as follows:

$$
\begin{aligned}
& \dot{M}(t)=\frac{1}{V_{s}}\left(-|\eta(t)| \sin (|\alpha(t)|)+A_{x} M^{2} \cos (|\alpha(t)|)\right) \\
& M(0)=3.0
\end{aligned}
$$

where $A_{x}=-1.96$ and $V_{s}=315.89 \mathrm{~m} / \mathrm{s}$ is the speed of sound at $20,000 \mathrm{ft}$, to provide a reasonably realistic Mach profile.

In a missile quasi-LPV model (1), the scheduled parameters are angle of attack $\alpha(t)$ and Mach number $M(t)$, which vary from $-25^{\circ}$ to $25^{\circ}$ and 2 to 4 , respectively. As the system (1) is symmetrical in terms of parameter $\alpha(t)$, only positive $\alpha(t)$ values need to be considered. Moreover, to reduce the conservatism on the following $H_{\infty}$ optimization controller design, the rate of scheduled parameter variation is also introduced. Following $\mathrm{Wu}$ et al., ${ }^{4)}$ the variation space of scheduled parameters is given as:

$$
\begin{aligned}
& \Theta=\{(\alpha, M): \alpha \in(0,25), M \in(2,4)\} \\
& \Psi=\{(\dot{\alpha}, \dot{M}): \dot{\alpha} \in(-200,200), \dot{M} \in(-0.5,0.5)\}
\end{aligned}
$$

Table 1. Missile model constants.

\begin{tabular}{ccc}
\hline Constants & Value & Unit \\
\hline$a_{n}$ & 0.000103 & $\mathrm{deg}^{-3}$ \\
$b_{n}$ & -0.00945 & $\mathrm{deg}^{-2}$ \\
$c_{n}$ & -0.1696 & $\mathrm{deg}^{-1}$ \\
$d_{n}$ & -0.034 & $\mathrm{deg}^{-1}$ \\
$a_{m}$ & 0.000215 & $\mathrm{deg}^{-3}$ \\
$b_{m}$ & -0.0195 & $\mathrm{deg}^{-2}$ \\
$c_{m}$ & 0.051 & $\mathrm{deg}^{-1}$ \\
$d_{m}$ & -0.206 & $\mathrm{deg}^{-1}$ \\
$K_{\alpha}$ & 1.18587 & $\mathrm{~s}^{-1}$ \\
$K_{q}$ & 70.586 & $\mathrm{~s}^{-2}$ \\
$K_{z}$ & 0.6661697 & \\
\hline
\end{tabular}

To realize TP transformation, the system in Eq. (1) is rewritten as

$$
\left(\begin{array}{l}
\dot{x}(t) \\
y(t)
\end{array}\right)=(S(\theta(t)))\left(\begin{array}{l}
x(t) \\
u(t)
\end{array}\right)
$$

with

$$
S(\theta(t))=\left(\begin{array}{ll}
A(\theta(t)) & B(\theta(t)) \\
C(\theta(t)) & D(\theta(t))
\end{array}\right) \in \mathbb{R}^{4 \times 3}
$$

where input is $u(t)=\delta(t)$, state vector is $x(t)=(\alpha(t), q(t))^{\mathrm{T}}$, the measured output is $y(t)=(\eta(t), q(t))^{\mathrm{T}}$ and scheduled parameters are $\theta(t)=(\alpha(t), M(t))$.

The autopilot specifications are given in terms of performance and robustness. There are two performance goals to be achieved: good tracking characteristic (P1), and small missile fin deflection rate (P2). The goal for robustness is mainly robust stability under aerodynamic coefficient perturbation. All of the specifications ${ }^{4,8)}$ for the missile autopilot are as follows:

P1: Track step commands in $\eta_{c}$ of various amplitudes with time constant $\tau \leq 0.35 \mathrm{~s}$ and overshoot $M_{p} \leq 10 \%$ and steady-state error $e \leq 1 \%$.

P2: The maximum tail deflection rate $\dot{\delta}_{\text {max }}$ for $1 \mathrm{~g}$ step command in $\eta_{c}$ should not exceed $25 \mathrm{deg} / \mathrm{s}$.

$\mathrm{R} 1$ : Robust stability is also guaranteed by varying $\pm 25 \%$ and $\pm 10 \%$ of angle of attack part and tail-deflection part in moment coefficients and force coefficients independently.

\subsection{Missile pitch-axis TP model}

The TP model transformation introduced in Barany ${ }^{11-14)}$ is a numerical method to transform the LPV dynamic model into a convex combination of LTI systems. Through TP model transformation, a system in the form of Eq. (5) can be approximately expressed as a parameter-varying convex combination of LTI system matrices $S_{i_{1}, i_{2}, \cdots, i_{N}}$ :

$$
\begin{aligned}
S(\theta(t)) & \approx \sum_{r=1}^{R} w_{r}(\theta(t)) S_{r} \\
& =\sum_{i_{1}=1}^{I_{1}} \sum_{i_{2}=1}^{I_{2}} \cdots \sum_{i_{n}=1}^{I_{n}} \prod_{n=1}^{N} w_{n, i_{n}}\left(\theta_{n}(t)\right) S_{i_{1}, i_{2}, \cdots, i_{N}} \\
& =\mathscr{S} \bigotimes_{n=1}^{N} w_{n}\left(\theta_{n}(t)\right)
\end{aligned}
$$

where $R=I_{1} \times I_{2} \times \cdots \times I_{N}$ is the number of the vertex systems, $N$ is the number of the scheduled parameters, and 
$w_{r}(\theta(t))$ are the weighting functions corresponding to vertex systems $S_{r}(t)$. The $(N+2)$ dimensional coefficient tensor $\mathscr{S} \in \mathbb{R}^{I_{1} \times I_{2} \times \cdots \times I_{N} \times O \times I}$ is constructed from the LTI vertex systems $S_{i_{1}, i_{2}, \cdots, i_{N}}$. The row vector $w_{n}\left(\theta_{n}(t)\right)$ contains one variable and continuous weighting functions $w_{n, i_{n}}\left(\theta_{n}(t)\right)$, $i_{n}=1, \cdots, I_{n}$. In this TP polytopic model representation, the weighting functions satisfy the following criteria

$$
\left\{\forall r, \theta(t): w_{r}(\theta(t)) \geq 0, \forall \theta(t): \sum_{r=1}^{R} w_{r}(\theta(t))=1\right\}
$$

and

$$
\begin{gathered}
\left\{\forall i, n, \theta_{n}(t): w_{n, i}\left(\theta_{n}(t)\right) \geq 0, \forall n, \theta_{n}(t):\right. \\
\left.\sum_{i_{n}=1}^{I_{N}} w_{n, i_{n}}\left(\theta_{n}(t)\right)=1\right\}
\end{gathered}
$$

For this typical LPV model, a large number of LMI-based controller design techniques can be immediately applied to develop the TP model. Another feature is to make a tradeoff between the modeling accuracy and computational complexity, which is useful for the complex system to reduce computation via a better low-rank approximation.

Now, TP model transformation is executed on the quasiLPV model (4). The grid density of the transformation space $\Theta$ defined in Eq. (3) is pointed out as $400 \times 200$. Through TP transformation, we receive 20 vertex LTI systems $S_{i_{1}, i_{2}} \in R^{4 \times 3}, i_{1}=1, \cdots, 5$ and $i_{2}=1, \cdots, 4$. But in view of the computational complexity, vertex systems $S_{i_{1}, i_{2}}$ can be reduced to $3 \times 3$. Comparing the TP polytopic model with the original quasi-LPV model (1), over 4,000 test points of randomly selected parameter values $(\alpha, M) \in \Theta$, the maximum and mean error obtained are 0.0087 and 0.0103 , respectively. This procedure which reduces complexity by half, can still ensure acceptable accuracy for real applications. Thus, it's reasonable to design a missile autopilot based on the missile pitch-axis TP model given as:

$$
\begin{aligned}
& {\left[\begin{array}{c}
\dot{\alpha} \\
\dot{q}
\end{array}\right] \approx \sum_{i_{1}=1}^{3} \sum_{i_{2}=1}^{3} w_{1, i_{1}}(\alpha) w_{2, i_{2}}(M)\left(A_{i_{1}, i_{2}}\left[\begin{array}{l}
\alpha \\
q
\end{array}\right]+B_{i_{1}, i_{2}} \delta\right)} \\
& {\left[\begin{array}{c}
\eta \\
q
\end{array}\right] \approx \sum_{i_{1}=1}^{3} \sum_{i_{2}=1}^{3} w_{1, i_{1}}(\alpha) w_{2, i_{2}}(M)\left(C_{i_{1}, i_{2}}\left[\begin{array}{l}
\alpha \\
q
\end{array}\right]+D_{i_{1}, i_{2}} \delta\right)}
\end{aligned}
$$

where the parameter-dependent weighting functions $w_{n, k}\left(\theta_{n}\right)$, $n=1,2, k=1, \cdots, 3$, are presented in Fig. 1 and the system matrices $A_{i_{1}, i_{2}}, B_{i_{1}, i_{2}}, C_{i_{1}, i_{2}}$ and $D_{i_{1}, i_{2}}$ are taken from the vertex system $S_{i_{1}, i_{2}}$ like Eq. (5). To check the precision of approximation from the quasi-LPV model to the TP polytopic system, the step responses of two systems are presented in Fig. 2. It is clear that the differences between the two response curves are fairly small. These results further illustrate the following analysis and controller design based on the TP polytopic model in Eq. (7) are feasible.

\section{State Feedback $\boldsymbol{H}_{\infty}$ Design with Pole Constraints Based on TP Polytopic Model}

Due to the advantages of the TP polytopic model for con-

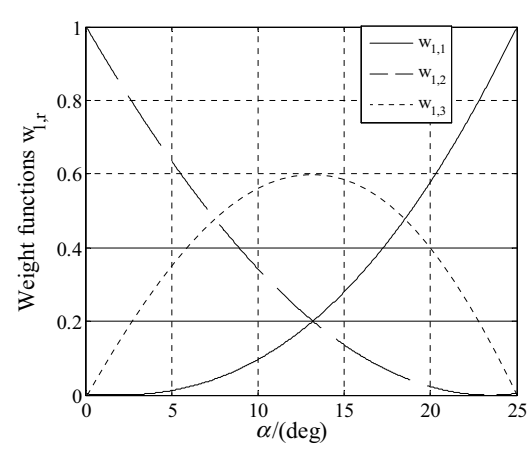

(a) Weight functions $w_{1, r}(\alpha)$

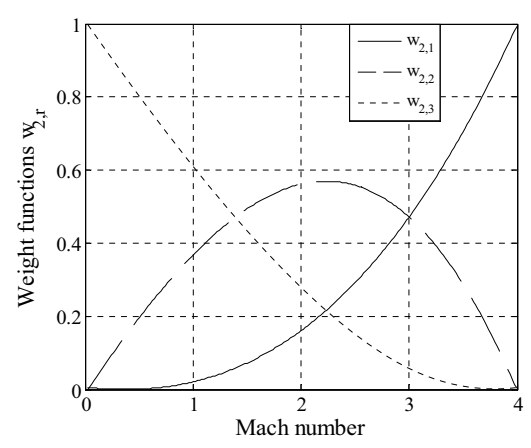

(b) Weight functions $w_{2, r}(M)$

Fig. 1. The TP weighting functions $w_{n, k}\left(\theta_{n}\right)$.

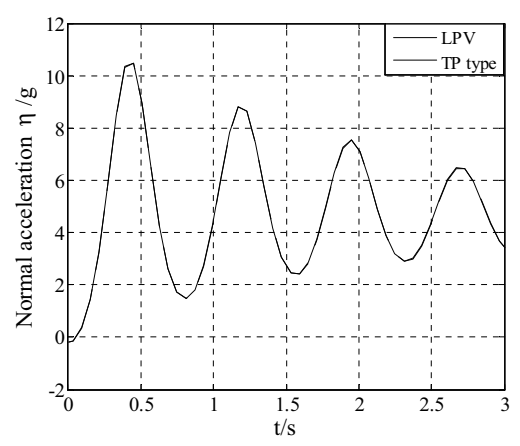

(a) Step responses of two models

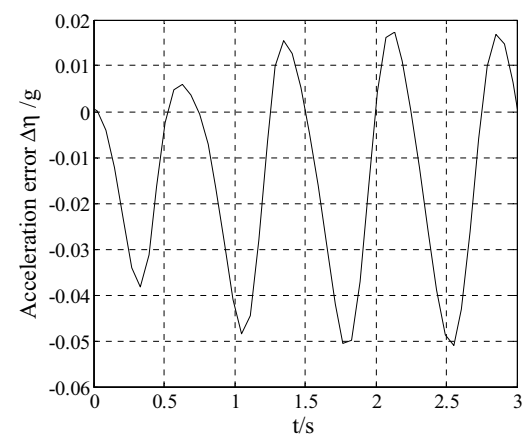

(b) Response error between two models

Fig. 2. The step response of the TP polytopic systems and quasi-LPV system.

vex hull manipulation, many LMI design methods ${ }^{15)}$ can be applied to LPV controller development. Such as in Baranyi et al., ${ }^{18)}$ the LMI performance specifications including 
asymptotic stability for the controller and observer, decay rate for controller and restrictions on the value of control signal are given based on the TP model. The design method for the TP model-based output feedback $H_{\infty}$ optimization is proposed in Chumalee and Whidborne. ${ }^{22)}$

In this paper, consider a LPV system described by

$$
\begin{aligned}
& \dot{x}(t)=A(\theta(t)) x(t)+B_{1}(\theta(t)) \omega(t)+B_{2}(\theta(t)) u(t) \\
& z(t)=C(\theta(t)) x(t)+D_{1}(\theta(t)) \omega(t)+D_{2}(\theta(t)) u(t)
\end{aligned}
$$

and let $T_{\omega z}(\theta)$ denote the closed-loop transfer function from $\omega(t)$ to $z(t)$ under state-feedback control $u(t)=K(\theta(t)) x(t)$. Now, our problem consists of finding a state-feedback gain $K(\theta(t))$ that:

- The poles of the closed-loop are placed in some LMI stability region $\mathcal{D}, \forall \theta \in \Theta$;

- Optimize $H_{\infty}$ performance.
Let $\left(A_{c l}(\theta), B_{c l}(\theta), C_{c l}(\theta), D_{c l}(\theta)\right)$ denote the state space realization of $T_{\omega z}(\theta)$, where

$$
\left(\begin{array}{ll}
A_{c l}(\theta) & B_{c l}(\theta) \\
C_{c l}(\theta) & D_{c l}(\theta)
\end{array}\right)=\left(\begin{array}{ll}
A(\theta)+B_{2}(\theta) K(\theta) & B_{1}(\theta) \\
C(\theta)+D_{2}(\theta) K(\theta) & D_{1}(\theta)
\end{array}\right)
$$

First, considering $H_{\infty}$ performance based on the parameter-dependent Lyapunov function ${ }^{22)} \quad V(x(t), \theta(t))=$ $x(t)^{\mathrm{T}} P(\theta(t)) x(t)$, the state feedback $H_{\infty}$ control problem to ensure parameter-dependent performance $\gamma(\theta)$ is equivalent to the existence of a symmetrical matrix $P(\theta)$ such that

$$
P(\theta)>0, \quad \frac{\mathrm{d}}{\mathrm{d} t}\left(x^{\mathrm{T}} P(\theta) x\right)+z^{\mathrm{T}} z-\gamma^{2} \omega^{\mathrm{T}} \omega<0
$$

along all possible parameter trajectories, $\forall(\theta, \dot{\theta}) \in \Theta \times \Phi$. Note that " $<0$ " stands for negative definite and " $>0$ " positive definite. The inequality (10) can yield

$$
\left(\begin{array}{ccc}
A_{c l}(\theta) X_{\infty}(\theta)+X_{\infty}(\theta) A_{c l}^{\mathrm{T}}(\theta)-\dot{X}_{\infty}(\theta) & B_{c l}(\theta) & X_{\infty}(\theta) C_{c l}^{\mathrm{T}}(\theta) \\
B_{c l}^{\mathrm{T}}(\theta) & -\gamma(\theta) I & D_{c l}^{\mathrm{T}}(\theta) \\
C_{c l}(\theta) X_{\infty}(\theta) & D_{c l}(\theta) & -\gamma(\theta) I
\end{array}\right)<0
$$

with $X_{\infty}(\theta)=P^{-1}(\theta)>0, \dot{X}_{\infty}(\theta)=\dot{P}^{-1}(\theta)=-P^{-1}(\theta) \dot{P}(\theta) \times P^{-1}(\theta)$. Let $X_{\infty}(\theta)$ and $\gamma(\theta)$ have the same TP polytopic structure of the model itself given as

$$
X_{\infty}(\theta)=\sum_{r=1}^{R} w_{r}(\theta) X_{r \infty}, \quad \gamma(\theta)=\sum_{r=1}^{R} w_{r}(\theta) \gamma_{r}
$$

Following Chumalee and Whidborne, ${ }^{22)}$ using an equation-error method leads to an approximate expression of $\dot{X}_{\infty}(\theta)$ as

$$
\dot{X}_{\infty}(\theta) \approx \sum_{k=1}^{M} \beta_{k}(\dot{\theta}) \tilde{X}_{k \infty}
$$

with

$$
\left\{\forall k, \dot{\theta}: \beta_{k}(\dot{\theta})>0, \forall \dot{\theta}: \sum_{k=1}^{M} \beta_{k}(\dot{\theta})=1\right\} \quad \text { and } \quad M=2^{N}
$$

Substituting Eqs. (7), (9), (12) and (13) into Eq. (11), it can be rewritten as

$$
\begin{gathered}
\sum_{r=1}^{R} \sum_{k=1}^{M} w_{r}^{2}(\theta) \beta_{k}(\dot{\theta})\left(\begin{array}{ccc}
-\tilde{X}_{k \infty}+\left(A_{r} X_{r \infty}+B_{2 r} L_{r \infty}+(*)\right) & B_{1 r} & X_{r \infty} C_{1 r}^{\mathrm{T}}+L_{r \infty}^{\mathrm{T}} D_{2 r}^{\mathrm{T}} \\
* & -\gamma_{r} I & D_{1 r}^{\mathrm{T}} \\
* & * & -\gamma_{r} I
\end{array}\right)+2 \sum_{i=1}^{R-1} \sum_{j=i+1}^{R} \sum_{k=1}^{M} w_{i}(\theta) w_{j}(\theta) \beta_{k}(\dot{\theta}) \frac{1}{2} \\
\times\left(\begin{array}{ccc}
-2 \tilde{X}_{k \infty}+\left(A_{i} X_{j \infty}+B_{2 i} L_{j \infty}+A_{j} X_{i \infty}+B_{2 j} L_{i \infty}+(*)\right) & B_{1 i}+B_{1 j} & X_{j \infty} C_{1 i}^{\mathrm{T}}+L_{j \infty}^{\mathrm{T}} D_{2 i}^{\mathrm{T}}+X_{i \infty} C_{1 j}^{\mathrm{T}}+L_{i \infty}^{\mathrm{T}} D_{2 j}^{\mathrm{T}} \\
* & -\gamma_{i} I-\gamma_{j} I & D_{1 i}^{\mathrm{T}}+D_{1 j}^{\mathrm{T}} \\
* & * & -\gamma_{i} I-\gamma_{j} I
\end{array}\right)<0
\end{gathered}
$$

where

$$
L_{\infty}(\theta)=K(\theta) X_{\infty}(\theta), \quad L_{\infty}(\theta)=\sum_{r=1}^{R} w_{r}(\theta) L_{r \infty}
$$

and the notation " $*$ " represents a symmetrical block.

Then the LMI pole constraints ${ }^{21)}$ based on the TP type polytopic model are taken in consideration like the above $H_{\infty}$ optimization. It is satisfied if, and only if, there exist matrices $X_{r 2}, r=1, \cdots, R$ such that

$$
\begin{gathered}
X_{2}(\theta)=\sum_{r=1}^{R} w_{r}(\theta) X_{r 2}>0 \\
\sum_{r=1}^{R} w_{r}^{2}(\theta)\left(\alpha \otimes X_{r 2}+\beta \otimes\left(A_{r} X_{r 2}+B_{2 r} L_{r 2}\right)\right. \\
\left.+\beta^{\mathrm{T}} \otimes\left(A_{r} X_{r 2}+B_{2 r} L_{r 2}\right)^{\mathrm{T}}\right) \\
+2 \sum_{i=1}^{R-1} \sum_{j=i+1}^{R} w_{i}(\theta) w_{j}(\theta) \frac{1}{2}\left(\alpha \otimes X_{i 2}+\beta \otimes\left(A_{j} X_{i 2}+B_{2 j} L_{i 2}\right)\right.
\end{gathered}
$$


where

$$
L_{2}(\theta)=K(\theta) X_{2}(\theta) \quad \text { and } \quad L_{2}(\theta)=\sum_{r=1}^{R} w_{r}(\theta) L_{r 2} .
$$

Note that " $\otimes$ " denotes the Kronecker product of matrices and that " $\alpha$ " and " $\beta$ " are symmetrical matrices to characterize the specified LMI region. ${ }^{21)}$

Our goal is to minimize $H_{\infty}$ performance $\left\|T_{\omega z}(\theta)\right\|_{\infty}$ over all state-feedback gains $K(\theta)$ that enforce the pole constraints. From the previous discussion, this is equivalent to minimizing $\gamma(\theta)$ over all matrices $X_{r 2}, X_{r \infty}, L_{r 2}$ and $L_{r \infty}$, $r=1, \cdots, R$ satisfying Eqs. (15) and (17). While the problem is not jointly convex in the variables $\left(X_{r 2}, X_{r \infty}\right.$, $\left.L_{r 2}, L_{r \infty}\right)$, convexity can be enforced by making

$$
X(\theta)=X_{2}(\theta)=X_{\infty}(\theta), \quad \forall \theta \in \Theta
$$

Simultaneously, since the LMI constraints (15)-(17) are affinely dependent on the scheduled parameters, a finite num-

$$
\begin{aligned}
& +\beta^{\mathrm{T}} \otimes\left(A_{j} X_{i 2}+B_{2 j} L_{i 2}\right)^{\mathrm{T}}+\alpha \otimes X_{j 2} \\
& \left.+\beta \otimes\left(A_{i} X_{j 2}+B_{2 i} L_{j 2}\right)+\beta^{\mathrm{T}} \otimes\left(A_{i} X_{j 2}+B_{2 i} L_{j 2}\right)^{\mathrm{T}}\right)<0
\end{aligned}
$$

ber of LMIs can be obtained according to the following lemma, which can easily be solved using existing software. The lemma taken from Gahinet et al. ${ }^{23)}$ is

Lemma 3.1: A given symmetrical matrix polytope, for which

$$
N(\theta)=\sum_{i=1}^{M} \alpha_{i}(\theta) \hat{N}_{i}
$$

with

$$
\left\{\forall i, \theta: \alpha_{i}(\theta) \geq 0, \forall \theta: \sum_{i=1}^{M} \alpha_{i}(\theta)=1\right\}
$$

as a negative symmetrical matrix for all possible parameter trajectories, $N(\theta)<0$, and $\forall \theta \in \Theta$ if, and only if, $\hat{N}_{i}<0$, $i=1, \cdots, M$.

Theorem 3.2: There exists an LPV state feedback controller $K(\theta)$ guaranteeing $H_{\infty}$ parameter-dependent performance $\gamma(\theta)$ of the closed-loop system in Eq. (9) with the $D$-stable constraints, $\forall(\theta, \dot{\theta}) \in \Theta \times \Phi$, if there exist some matrices, $X_{r}, L_{r}, r=1, \cdots, R$, satisfying Eqs. (19)-(23).

$$
X_{r}>0
$$

$$
\begin{gathered}
\alpha \otimes X_{i}+\beta \otimes\left(A_{j} X_{i}+B_{2 j} L_{i}\right)+\beta^{\mathrm{T}} \otimes\left(A_{j} X_{i}+B_{2 j} L_{i}\right)^{\mathrm{T}}+\alpha \otimes X_{j}+\beta \otimes\left(A_{i} X_{j}+B_{2 i} L_{j}\right)+\beta^{\mathrm{T}} \otimes\left(A_{i} X_{j}+B_{2 i} L_{j}\right)^{\mathrm{T}}<0 \\
\left(\begin{array}{ccc}
-\tilde{X}_{k}+\left(A_{r} X_{r}+B_{2 r} L_{r}+(*)\right) & B_{1 r} & X_{r} C_{1 r}^{\mathrm{T}}+L_{r}^{\mathrm{T}} D_{2 r}^{\mathrm{T}} \\
* & -\gamma_{r} I & D_{1 r}^{\mathrm{T}} \\
* & * & -\gamma_{r} I
\end{array}\right)<0 \\
\left(\begin{array}{ccc}
-2 \tilde{X}_{k}+\left(A_{i} X_{j}+B_{2 i} L_{j}+A_{j} X_{i}+B_{2 j} L_{i}+(*)\right) & B_{1 i}+B_{1 j} & X_{j} C_{1 i}^{\mathrm{T}}+L_{j}^{\mathrm{T}} D_{2 i}^{\mathrm{T}}+X_{i} C_{1 j}^{\mathrm{T}}+L_{i}^{\mathrm{T}} D_{2 j}^{\mathrm{T}} \\
* & -\gamma_{i} I-\gamma_{j} I & D_{1 i}^{\mathrm{T}}+D_{1 j}^{\mathrm{T}} \\
* & * & -\gamma_{i} I-\gamma_{j} I
\end{array}\right)<0
\end{gathered}
$$

for $r=1, \cdots, R, k=1, \cdots, M$ and $1 \leq i<j \leq R$.

Proof: By applying lemma 3.1 to LMI constraints Eqs. (15)-(17) with the additional condition Eq. (18), theorem 3.2 can be obtained.

Note that matrices $\tilde{X}_{k}, k=1, \cdots, M$ in Eqs. (22) and (23) can be transformed into the combinations of $X_{r}, r=1, \cdots$, $R$, which do not increase the number of variables. For optimizing $H_{\infty}$ norm, we reasonably minimize the index $1 / R \sum_{r=1}^{R} \gamma_{r}$ to achieve this goal. Through the existing software YALMIP parser ${ }^{24)}$ and SEDUMI solver, ${ }^{25)}$ these LMIs are capable of being solved numerically. Now, a new gainscheduling method based on the TP polytopic model is proposed for designing a parameter-dependent state-feedback controller to minimize the $H_{\infty}$ performance of the closedloop system that enforced LMI pole limits.

\section{Autopilot Design}

\subsection{Controller development}

A simple controller architecture means low expense and easy to implement, so engineers always avoid using the complex high-order dynamic missile autopilot in practice. This paper introduces the novel four-loop autopilot structure ${ }^{7,26)}$ shown in Fig. 3, which just requires to design four scheduled parameters $K_{e}, K_{a}, K_{\omega}$ and $K_{\delta}$. For facilitating controller development, this structure can be rearranged as the LFT type in Fig. 4. One can see that the generalized perturbation signal $\omega$ is the reference normal acceleration $\eta_{c}$, the performance in$\operatorname{dex} z$ is acceleration tracking error $e$, the measured output is

$$
y=\left(\int e, \eta, q, \delta\right)^{\mathrm{T}}
$$

and the controller output $u$ is actuator command $\delta_{c}$.

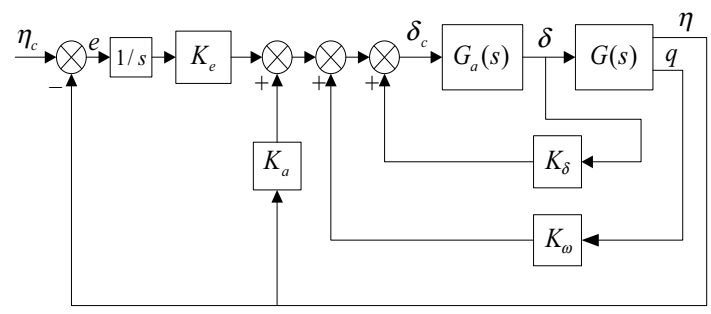

Fig. 3. A four-loop autopilot structure. 
In open-loop controller development, a first-order lag actuator $G_{a}(s)$ is introduced as

$$
\dot{\delta}=\tau\left(\delta_{c}-\delta\right)
$$

where $\tau=150 \mathrm{~Hz}$. Combined with the missile pitch-axis TP model Eq. (7), the generalized model of open-loop structure in Fig. 4 is obtained in the following fashion ${ }^{7)}$ :

$$
\begin{aligned}
& {\left[\begin{array}{c}
\dot{x} \\
z \\
y
\end{array}\right]=\left[\begin{array}{ccc}
A(\theta) & B_{\omega}(\theta) & B_{u}(\theta) \\
C_{z}(\theta) & D_{z \omega}(\theta) & D_{z u}(\theta) \\
C_{y}(\theta) & D_{y \omega}(\theta) & D_{y u}(\theta)
\end{array}\right]\left[\begin{array}{l}
x \\
\omega \\
u
\end{array}\right]} \\
& =\sum_{i_{1}=1}^{3} \sum_{i_{2}=1}^{3} w_{1, i_{1}}(\alpha) w_{2, i_{2}}(M)\left[\begin{array}{ccc}
A_{i_{1}, i_{2}} & B_{\omega_{i_{1}, i_{2}}} & B_{u_{i_{1}, i_{2}}} \\
C_{z_{i_{1}, i_{2}}} & 1 & 0 \\
C_{y_{i_{1}, i_{2}}} & 0_{4 \times 1} & 0_{4 \times 1}
\end{array}\right]\left[\begin{array}{c}
x \\
\omega \\
u
\end{array}\right]
\end{aligned}
$$

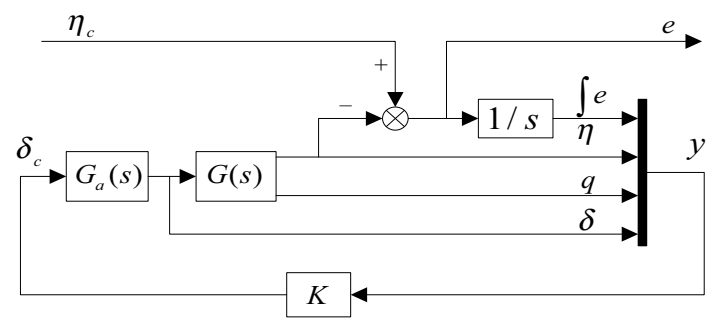

Fig. 4. Open-loop controller synthesis diagram.

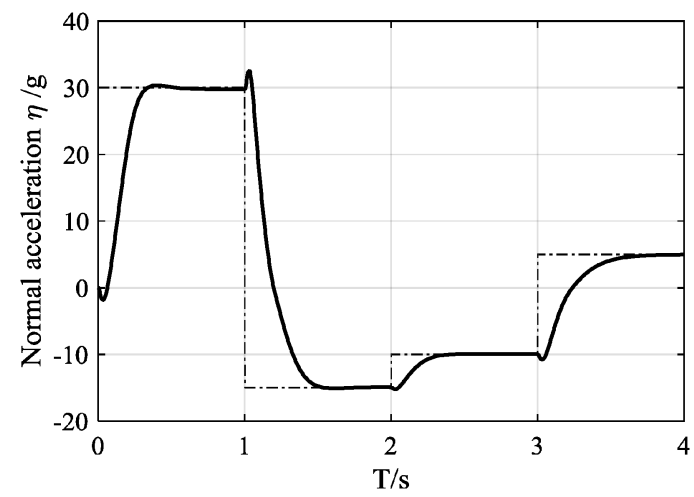

(a) Normal acceleration

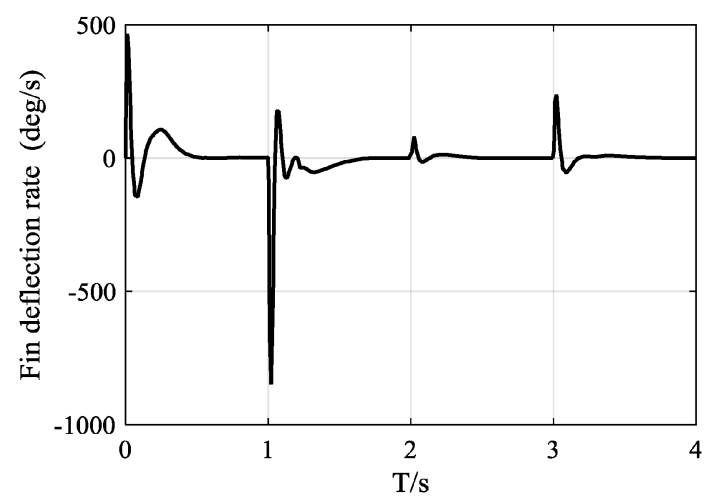

(c) Fin deflection rate
Due to the fact that the measurement matrix $C_{y}(\theta)$ is of full rank, $\forall \theta \in \Theta$, the output feedback controller $K_{y}(\theta)$ can be computed according to the state-feedback controller $K_{x}(\theta)$ via the following relation:

$$
K_{y}(\theta)=K_{x}(\theta) C_{y}^{-1}(\theta)
$$

A LMI region $(\lambda, \omega, \xi)$ is imposed for pole clustering, with $\lambda=9, \omega=100$ and $\xi=0.80$ being the minimum decay rate, maximum undamped natural frequency and minimum damping ratio, respectively. Now, we can apply theorem 3.2 to receive a controller that optimizes the $H_{\infty}$ performance of the closed-loop system in Eq. (25) that enforced pole constraints. The with $\lambda=9, \omega=100$ and $\xi=0.80$ being the minimum decay rate, maximum undamped natural frequency and minimum damping ratio, respectively. Now, we can apply theorem 3.2 to receive a controller that optimizes the $H_{\infty}$ performance of the closed-loop system in Eq. (25) that enforced pole constraints. The computational time for solving these LMIs is less than one minute on a regular computer.

\subsection{Simulation results}

As angle of attack $\alpha$ is not directly measurable in real-time, an approximation ${ }^{4)}$ of $\alpha$ is given from the measured output by

$$
\begin{aligned}
\alpha_{e}= & -1.396-0.33421 M_{N}-3.7653 \delta_{N}-0.91681 \delta_{N} M_{N} \\
& +\eta_{N}\left(-46.03+21.26 M_{N}-8.8362 M_{N}^{2}-0.33564 \delta_{N}\right. \\
& \left.+0.385 \delta_{N} M_{N}+0.32892 \delta_{N} M_{N}^{2}\right)
\end{aligned}
$$

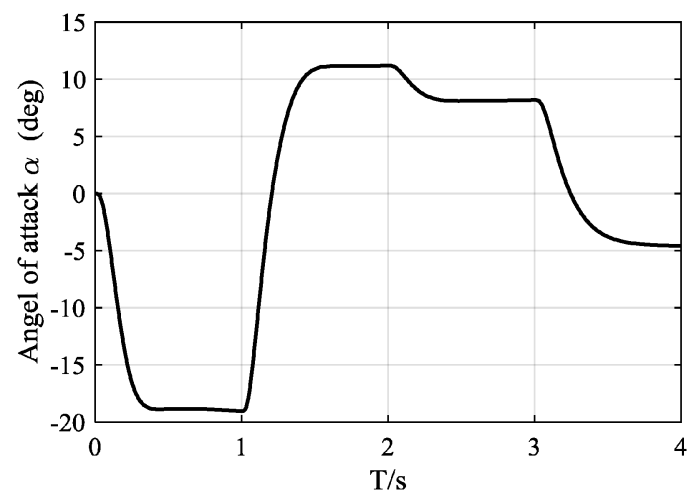

(b) Angle of attack

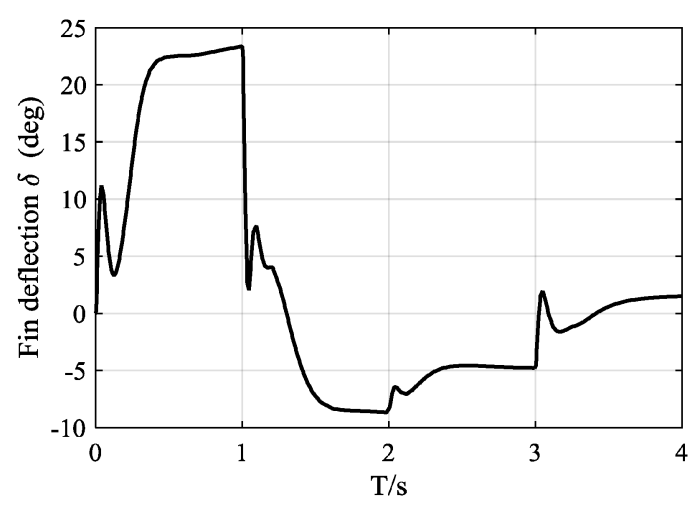

(d) Fin deflection

Fig. 5. Close-loop response in a nominal situation. 


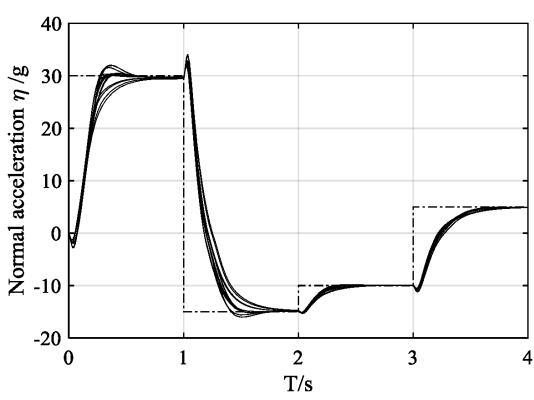

(a) Normal acceleration

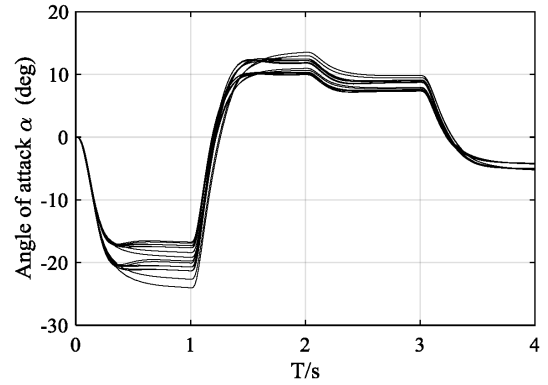

(b) Angle of attack

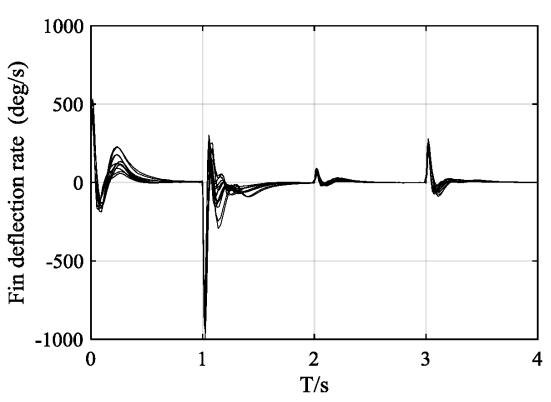

(c) Fin deflection rate

Fig. 6. Close-loop response under perturbation.

$$
\begin{aligned}
& +\eta_{N}^{3}\left(61.367-69.756 M_{N}+30.44 M_{N}^{2}+3.9589 \delta_{N}\right. \\
& \left.-15.668 \delta_{N} M_{N}+11.498 \delta_{N} M_{N}^{2}\right) \\
& +\eta_{N}^{5}\left(-54.655+94.381 M_{N}-48.212 M_{N}^{2}-4.7973 \delta_{N}\right. \\
& \left.+18.807 \delta_{N} M_{N}-13.871 \delta_{N} M_{N}^{2}\right)
\end{aligned}
$$

where $\eta_{N}=\eta / 60, \delta_{N}=(\delta-10) / 25$ and $M_{N}=M-3.0$.

First, the proposed autopilot is simulated at the nominal values of missile aerodynamic coefficients. The scope of this simulation is to demonstrate fulfillment of the performance specifications, P1 and P2 from Sec. 2. Tracking performance of normal acceleration $\eta(t)$ in response to a typical amplitude-varying reference command $\eta_{c}(t)$ is presented in Fig. 5(a). The corresponding angle of attack $\alpha(t)$, fin deflection rate $\dot{\delta}(t)$ and fin deflection $\delta(t)$ are plotted in Figs. 5(b)(d), respectively. It can be seen that the performance goals are satisfied. Compared with the former LPV controllers, ${ }^{4,22)}$ the results are similar, while the controller structure we utilized is simpler.

In order to assess the robustness of the missile autopilot, moment coefficients and force coefficients ${ }^{4,6)}$ are perturbed independently. For moment coefficients $a_{m}, b_{m}$ and $c_{m}$ are simultaneously changed from their nominal values by $\pm 25 \%$ and $d_{m}$ is changed by $\pm 25 \%$. Similar perturbations are imposed on force coefficients, but are limited to $\pm 10 \%$. The combination of all these variations leads to 16 different plots in Figs. 6(a)-(c). As one can see, stability is maintained in all cases and the performance is still satisfactory or reasonably degradated.

\section{Conclusion}

In this article, a novel tensor product model-based gainscheduling technique is successfully applied to design a pitch-axis autopilot for air-to-air missiles. This approach could optimize the $H_{\infty}$ performance of the closed-loop system enforced LMI pole constraints. The simulation results obtained illustrate that the stability and performance robustness specifications are met even if a simple controller architecture is retained, and verify the effectiveness of our presented method. Compared with other existing TP-based LMI methods, this technique provides a more flexible and efficient process to achieve the specification requirements by choosing different LMI regions to bound the poles.

\section{References}

1) Rugh, W. J. and Shamma, J. S.: Research on Gain Scheduling, Automatica, 36 (2000), pp. 1401-1425.

2) Shamma, J. S. and Cloutier, J. R.: Gain-scheduled Missile Autopilot Design using Linear Parameter Varying Transformations, J. Guid. Control Dynam., 16 (1993), pp. 256-263.

3) Pellanda, P. C., Apkarian, P., and Tuan, H. D.: Missile Autopilot Design via a Multi-channel LFT/LPV Control Method, Int. J. Robust Nonlin., 12 (2002), pp. 1-20.

4) Wu, F., Packard, A., and Balas, G.: Systematic Gain-Scheduling Control Design: A Missile Autopilot Example, Asian J. Contro., 4 (2002), pp. 341-347.

5) Stilwell, D. J.: State-space Interpolation for a Gain-scheduled Autopilot, J. Guid. Control Dynam., 24 (2001), pp. 460-465.

6) Theodoulis, S. and Duc, G.: Missile Autopilot Design: Gain-scheduling and the Gap Metric, J. Guid. Control Dynam., 32 (2009), pp. 986-996.

7) Theodoulis, S., Gassmann, V., Wernert, P., Dritsas, L., Kitsios, I., and Tzes, A.: Guidance and Control Design for a Class of Spin-stabilized Fin-controlled Projectiles, J. Guid. Control Dynam., 36 (2013), pp. 517-531.

8) Apkarian, P.: On the Discretization of LMI-synthesized Linear Parameter-varying Controllers, Automatica, 33 (1997), pp. 655-661.

9) White, B. A., Bruyere, L., and Tsourdos, A.: Missile Autopilot Design using Quasi-LPV Polynomial Eigenstructure Assignment, IEEE T. Aero. Elec. Sys., 43 (2007), pp. 1470-1483.

10) Apkarian, P. and Adams, R. J.: Advanced Gain-scheduling Techniques for Uncertain Systems, IEEE T. Contr. Syst. T., 6 (1998), pp. 21-32.

11) Baranyi, P.: TP Model Transformation as a Way to LMI-based Controller Design, IEEE T. Ind. Electron., 51 (2004), pp. 387-400.

12) Baranyi, P.: Tensor Product Model-based Control of Two-dimensional Aeroelastic System, J. Guid. Control Dynam., 29 (2006), pp. 391-400.

13) Baranyi, P.: Output Feedback Control of Two-Dimensional Aeroelastic System, J. Guid. Control Dynam., 29 (2006), pp. 762-767.

14) Baranyi, P.: The Generalized TP Model Transformation for T-S Fuzzy Model Manipulation and Generalized Stability Verification, IEEE T. Fuzzy Syst., 22 (2014), pp. 934-948.

15) Boyd, S., Ghaoui, L. E., Feron, E., and Balakrishnan, V.: Linear Matrix Inequalities in System and Control Theory, SIAM, Philadelphia, 1994.

16) Takarics, B. and Baranyi, P.: Tensor-product-model-based Control of a Three Degrees-of-freedom Aeroelastic Model, J. Guid. Control Dynam., 36 (2013), pp. 1527-1533.

17) Baranyi, P. and Takarics, B.: Aeroelastic Wing Section Control via Relaxed Tensor Product Model Transformation Framework, J. Guid. Control Dynam., 37 (2014), pp. 1671-1678.

18) Baranyi, P., Yam, Y., and Varlaki, P.: Tensor Product Model Transformation in Polytopic Model-based Control, CRC Press, Florida, 2013.

19) Baranyi, P., Korondi, P., and Tanaka, K.: Parallel Distributed Compensation Based Stabilization of a 3-DOF RC Helicopter: A Tensor Product Transformation Based Approach, Int. J. Comput. Int. Sys., 13 (2009), pp. 25-34. 
Trans. Japan Soc. Aero. Space Sci., Vol. 59, No. 3, 2016

20) Precup, R., Dragos, C., Preitl, S., and Petriu, E. M.: Novel Tensor Product Models for Automatic Transmission System Control, IEEE Syst. J., 6 (2012), pp. 488-498.

21) Chilali, M. and Gahinet, P.: $H_{\infty}$ Design with Pole Placement Constraints: An LMI Approach, IEEE T. Automat. Contr., 41 (1996), pp. 358-367.

22) Chumalee, S. and Whidborne, J. F.: Gain-Scheduled $H_{\infty}$ Control for Tensor Product Type Polytopic Plants, Asian J. Control, 17 (2015), pp. 417-431.

23) Gahinet, P., Apkarian, P., and Chilali, M.: Affine Parameter-dependent Lyapunov Functions and Real Parametric Uncertainty, IEEE T. Automat. Contr., 41 (1996), pp. 436-442.

24) Lofberg, J.: Yalmip: A Toolbox for Modeling and Optimization in MATLAB, Computer Aided Control Systems Design, 2004 IEEE In- ternational Symposium on Computer Aided Control Systems Design, Taipei, Taiwan, 2004.

25) Sturm, J. F.: Using SeDuMi 1.02, A Matlab Toolbox for Optimization over Symmetric Cones, Optim. Method Softw., 11 (2008), pp. 625-653.

26) Mracek, C. P. and Ridgely, D. B.: Missile Longitudinal Autopilots: Connections between Optimal Control and Classical Topologies, AIAA Guidance, Navigation and Control Conference and Exhibit, San Francisco, California, 2005.

Y. Ochi

Associate Editor 